International Conference Mathematical and Computational Biology 2011

International Journal of Modern Physics: Conference Series

Vol. 9 (2012) 259-270

C World Scientific Publishing Company

DOI: $10.1142 / \mathrm{S} 2010194512005302$

\title{
SOFT SET-BASED DECISION MAKING FOR PATIENTS SUSPECTED INFLUENZA-LIKE ILLNESS
}

\author{
TUTUT HERAWAN \\ Database and Knowledge Management Research Group \\ Faculty of Computer System and Software Engineering \\ Universiti Malaysia Pahang \\ Lebuh Raya Tun Razak, Gambang 26300, Kuantan, Pahang, Malaysia \\ tutut@ump.edu.my
}

\begin{abstract}
In previous work, we presented an applicability of soft set theory for decision making of patients suspected influenza. The proposed technique is based on maximal supported objects by parameters. At this stage of the research, results are presented and discussed from a qualitative point of view against recent soft decision making techniques through an artificial dataset. In this paper, we present an extended application of our soft set-based decision making through a Boolean valued information system from a dataset of patients suspected ILI (Influenza-Like Illness). Using soft set theory and maximal symptoms co-occurences in patients, we explore how soft set-based decision making technique can be used to reduce the number of dispensable symptoms and further make a correct and fast decision. The result of this work can be used for recommendation of decision making based on the clusters decision captured. Finally, this technique may potentially contribute to lowering the complexity of medical decision making without loss of original information.
\end{abstract}

Keywords: Decision making; soft set theory; ILI dataset.

\section{Introduction}

An influenza pandemic is an epidemic of an influenza virus that spreads on a worldwide scale and infects a large proportion of the human population. The World Health Organization (WHO) and the U.S. Centers for Disease Control and Prevention (CDC) have been warning that there is a substantial risk of an influenza pandemic within the next few years [1]. Since the first case of novel influenza infection was announced in Mexico in April 2009, swH1N1 Influenza A- a mosaic of swine/bird/human Influenza A1- has spread across the world at lightning speed. This is likely to be the tip of the iceberg since surveillance systems are not widespread in any country [2]. Many healthcare leaders find themselves overwhelmed with data [3]. Another hand, a lot of work on influenza modeling has been done all over the world over the past 30 years based on the infectious diseases data including [4,5,6,7,8]. Recently, Karim and Razali proposed Mathematical Model of Influenza A, H1N1 for Malaysia [9]. They study the development of mathematical model to help understand the dynamics of an epidemic, to design treatment and develop a control strategie such as a vaccination program or quarantine policy. However, the works mentioned above lack the information they need 
to make informed decisions. One aspect that medical database plays an important role is in the field of decision making. Inputs obtained from dataset are stored in terms of records and attributes in databases do contribute a lot in the process of decision making. To this, one practical problem is faced: for a particular property, whether all the attributes in the attributes set are always necessary to preserve this property [10]. Computational models of the artificial intelligence such as soft set theory [11] have several applications. Parameterization reduction under soft set theory $[12,13,14,15,16,17]$ can be considered further as a technique for medical decision making. One possible application is the medical decision making of patients suspected Influenza-Like Illness (ILI).

In previous work, we presented an applicability of soft set theory for decision making of patients suspected influenza [18]. The proposed technique is based on maximal supported objects by parameters. We note that the soft set is designed to replace a Boolean-valued information system [19]. The research described in the paper is a part of our short term effort in applying soft set theory in order to make a decision and further grouping of patients under certain symptoms of influenza. At this stage of the research, results are presented and discussed from a qualitative point of view against recent soft decision making techniques through an artificial dataset.

In this paper, we present an extended application of our soft set-based decision making through a Boolean valued information system from a dataset of patients suspected Influenza-Like Illness (ILI). Since soft set theory is not yet widely known, we start from a tutorial introduction. Further the concept of soft dimensionality reduction and decision making techniques [16,18] are described. Using soft set theory and maximal symptoms co-occurences in patients, we explore how soft set-based decision making technique can be used to reduce the number of dispensable symptoms and further make a correct and fast decision. The result of this work can be used for recommendation of decision making based on the clusters decision captured. Finally, this technique may potentially contribute to lowering the complexity of medical decision making without loss of original information.

The rest of this paper is organized as follows. Section 2 describes the notion of information system (databases). Section 3 describes the theory of soft set. Section 4 describes the dataset, modeling process and soft set-based decision making using maximal supported objects by parameters. Section 5 describes the results from an application of soft set theory for decision making and grouping patients suspected Influenza-Like Illness (ILI) following by discussion. Finally, the conclusion of this work is described in section 6 .

\section{Information System}

The syntax of information systems is very similar to relations in relational databases. Entities in relational databases are also represented by tuples of attribute values. An information system is a quadruple $S=(U, A, V, f)$, where $U=\left\{u_{1}, u_{2}, \cdots, u_{|U|}\right\}$ is a nonempty finite set of objects, $A=\left\{a_{1}, a_{2}, \cdots, a_{|A|}\right\}$ is a non-empty finite set of attributes, $V=\bigcup_{a \in A} V_{a}, V_{a}$ is the domain (value set) of attribute $a, f: U \times A \rightarrow V$ is an information function such that $f(u, a) \in V_{a}$, for every $(u, a) \in U \times A$, called information (knowledge) function [19]. An information system is also called a knowledge 
representation systems or an attribute-valued system. In an information system $S=(U, A, V, f)$, if $V_{a}=\{0,1\}$, for every $a \in A$, then $S$ is called a Boolean-valued information system. In many medical information systems, there is an outcome of classification that is known. This a posteriori knowledge is expressed by one (or more) distinguished attribute called decision attribute; the process is known as supervised learning. An information system of this kind is called a decision system. A decision system is an information system of the form $D=(U, A \cup\{d\}, V, f)$, where $d \notin A$ is the decision attribute [17]. The elements of $A$ are called condition attributes. A simple example of a decision table can be found in Table 1.

Example 1. (See [18]). A decision system of six patients with six symptoms (conditions) and a decision.

Table 1. An example of a decision system

\begin{tabular}{cccccccc}
\hline $\boldsymbol{U}$ & Fever & Cough & $\begin{array}{c}\text { Runny } \\
\text { Nose }\end{array}$ & Lethargic & Tired & Headache & Flu \\
\hline$p_{1}$ & yes & yes & no & yes & no & yes & yes \\
$p_{2}$ & no & no & yes & no & yes & no & no \\
$p_{3}$ & yes & yes & no & yes & no & yes & yes \\
$p_{4}$ & yes & no & no & no & yes & no & no \\
$p_{5}$ & yes & yes & yes & no & no & yes & yes \\
$p_{6}$ & no & no & no & yes & yes & no & no \\
\hline
\end{tabular}

\section{Soft Set Theory}

Throughout this section $U$ refers to an initial universe, $E$ is a set of parameters, $P(U)$ is the power set of $U$.

Definition 1. (See [11].) A pair $(F, E)$ is called a soft set over $U$, where $F$ is a mapping given by

$$
F: E \rightarrow P(U)
$$

In other words, a soft set over universe $U$ is a parameterized family of subsets of universe $U$. For $e \in E, F(e)$ may be considered as the set of $e$-elements of the soft set $(F, E)$ or as the set of $e$-approximate elements of the soft set. Clearly, a soft set is not a (crisp) set.

Example 2. (See [18]). Let we consider a soft set $(F, E)$ which describes the "conditions of patients suspected influenza" that a hospital is considering to make a decision. The six influenza symptoms, i.e., fever, respiratory, nasal discharges, cough, headache and sore throat are adopted from [20] and one symptom added is lethargic. Suppose that there are six patients in the hospital under consideration, 


$$
U=\left\{h_{1}, h_{2}, h_{3}, h_{4}, h_{5}, h_{6}\right\}
$$

and $E$ is a set of decision parameters

$$
E=\left\{e_{1}, e_{2}, e_{3}, e_{4}, e_{5}, e_{6}, e_{7}\right\}
$$

where

$e_{1}$ stands for the parameter "fever",

$e_{2}$ stands for the parameter "respiratory",

$e_{3}$ stands for the parameter "nasal discharges",

$e_{4}$ stands for the parameter "cough",

$e_{5}$ stands for the parameter "headache"

$e_{6}$ stands for the parameter "sore throat"

$e_{7}$ stands for the parameter "lethargic".

Consider the mapping $F: E \rightarrow P(U)$ given by "patients (.)", where (.) is to be filled in by one of parameters $e \in E$. Suppose that

$$
\begin{aligned}
& F\left(e_{1}\right)=\left\{p_{1}, p_{3}, p_{4}, p_{5}, p_{6}\right\}, \\
& F\left(e_{2}\right)=\left\{p_{1}, p_{2}\right\}, \\
& F\left(e_{3}\right)=\left\{p_{1}, p_{2}, p_{4}\right\}, \\
& F\left(e_{4}\right)=\left\{p_{1}\right\}, \\
& F\left(e_{5}\right)=\left\{p_{3}, p_{4}\right\}, \\
& F\left(e_{6}\right)=\left\{p_{2}, p_{4}\right\}, \\
& F\left(e_{7}\right)=\left\{p_{1}, p_{3}, p_{5}, p_{6}\right\} .
\end{aligned}
$$

Therefore, $F\left(e_{1}\right)$ means "patients suffer fever", whose functional value is the set $\left\{p_{1}, p_{3}, p_{4}, p_{5}, p_{6}\right\}$. Thus, we can view the soft set $(F, E)$ as a collection of approximations as below

$$
(F, E)=\left\{\begin{array}{c}
\text { fever }=\left\{p_{1}, p_{3}, p_{4}, p_{5}, p_{6}\right\}, \\
\text { respiratory }=\left\{p_{1}, p_{2}\right\}, \\
\text { nasal discharges }=\left\{p_{1}, p_{2}, p_{4}\right\}, \\
\text { cough }=\left\{p_{1}\right\}, \\
\text { head ache }=\left\{p_{3}, p_{4}\right\}, \\
\text { sore throat }=\left\{p_{2}, p_{4}\right\}, \\
\text { lethargic }=\left\{p_{1}, p_{3}, p_{5}, p_{6}\right\}
\end{array}\right\}
$$


Table 2. Tabular representation of a soft set in the above example

\begin{tabular}{cccccccc}
\hline $\boldsymbol{U}$ & $e_{1}$ & $e_{2}$ & $e_{3}$ & $e_{4}$ & $e_{5}$ & $e_{6}$ & $e_{7}$ \\
\hline$p_{1}$ & 1 & 1 & 1 & 1 & 0 & 0 & $\mathbf{1}$ \\
$p_{2}$ & 0 & 1 & 1 & 0 & 0 & 1 & $\mathbf{0}$ \\
$p_{3}$ & 1 & 0 & 0 & 0 & 1 & 0 & $\mathbf{1}$ \\
$p_{4}$ & 1 & 0 & 1 & 0 & 1 & 1 & $\mathbf{0}$ \\
$p_{5}$ & 1 & 0 & 0 & 0 & 0 & 0 & $\mathbf{1}$ \\
$p_{6}$ & 1 & 0 & 0 & 0 & 0 & 0 & $\mathbf{1}$ \\
\hline
\end{tabular}

Each approximation has two parts, a predicate $e$ and an approximate value set $p$. For example, for the approximation "fever $=\left\{p_{1}, p_{3}, p_{4}, p_{5}, p_{6}\right\}$ ", we have the predicate name of patients with fever and its value set is $\left\{p_{1}, p_{3}, p_{4}, p_{5}, p_{6}\right\}$.

The relation between a soft set and a Boolean-valued information system is given in the following proposition.

Proposition 1. (See [19]). If $(F, E)$ is a soft set over the universe $U$, then $(F, E)$ is a binary-valued information system $S=\left(U, A, V_{\{0,1\}}, f\right)$.

Proof. Let $(F, E)$ be a soft set over the universe $U$, we define a mapping

$$
F=\left\{f_{1}, f_{2}, \cdots, f_{n}\right\}
$$

where

$$
f_{i}: U \rightarrow V_{i} \text { and } f_{i}(x)=\left\{\begin{array}{ll}
1, & x \in F\left(e_{i}\right) \\
0, & x \notin F\left(e_{i}\right)
\end{array} \text {, for } 1 \leq i \leq|A|\right.
$$

Hence, if $A=E, V=\bigcup_{e_{i} \in A} V_{e_{i}}$, where $V_{e_{i}}=\{0,1\}$, then a soft set $(F, E)$ can be considered as a binary-valued information system $S=\left(U, A, V_{\{0,1\}}, f\right)$.

From Proposition 1, it is easily to understand that a binary-valued information system can be represented as a soft set. Thus, we can make a one-to-one correspondence between $(F, E)$ over $U$ and $S=\left(U, A, V_{\{0,1\}}, f\right)$.

\section{Material and Method}

The second part of this article starts from a description of the Influenza-Like Illness (ILI) dataset and the medical problem for which the data was collected. This work will identify which symptoms are dispensable and further can be removed from dataset without 
affected the original decision. Then, we make a decision of the reduced dataset based on the clusters captured.

\subsection{Material}

The current flu pandemic caused by the Influenza Type A-virus started in late March 2009 and to date has infected more than 1 million persons all over the world with more than 15,000 deaths in 182 countries. Malaysia is one of the countries has registered about 12,000 cases with 77 fatalities [9]. In this work, the data of patients suspected InfluenzaLike Illness (ILI) was collected from University Medical Center, Universiti Tun Hussein Onn Malaysia [21] during May-August 2009 with the permission of the center head. The dataset is summarized in Table 3. There are 182 suspects of Influenza-Like Illness and five symptoms founded; fever ( $>38$ degrees Celsius), cough, difficult breathing, tired and lethargic. All attributes are Boolean-valued, signifying the absence or presence of some feature. If $f(u, a)=1$, then the symptom is occur and otherwise is not. The following table summarized the data.

Table 3. A summary of the ILI dataset

\begin{tabular}{lllccc}
\hline Month & Fever & Cough & $\begin{array}{c}\text { Difficult } \\
\text { Breathing }\end{array}$ & Tired & Lethargic \\
\hline May & 6 & 5 & 1 & 2 & 0 \\
June & 2 & 2 & 0 & 1 & 0 \\
July & 63 & 61 & 1 & 6 & 2 \\
August & 111 & 104 & 7 & 9 & 1 \\
\hline
\end{tabular}

The problem of the soft set based decision making trough the above dataset is how to clusters the suspects of Influenza-Like Illness (ILI) in the same group with similar characteristics (symptoms). It can be done, firstly among all attributes we want to remove dispensable attributes. To select the candidate of the dispensable attributes, we employ the method proposed by $[16,18]$. Lastly, for capturing the decision (clusters) of suspect we refers to the cleansed data and group the suspects having the same symptoms.

\subsection{Modeling Process}

We turn now to the short discussion of the modeling for decision making process. The process has three basic steps which are briefly discussed.

a. Transformation. It follows from Proposition 3, in using soft set thery, the dataset is transformed into a Boolean-valued information system $S=\left(U, A, V_{\{0,1\}}, f\right)$.

b. Reduction. Before make a decision, the dataset must be cleaned first by removing dispensable symptoms. Here, we use the concept of soft set based-reduction as proposed in $[16,18]$.

c. Decision making. Applying the proposed method to make a decision (to classify) patients.

\subsection{Method}

The problem of parameterization reduction and decision making through this view is to reduce the number of parameter (symptoms) which preserved the consistence of optimal and sub-optimal decisions $[16,18]$. Throughout this sub-section the pair $(F, E)$ refers to 
the soft set over the universe $U$ representing a Boolean-valued information system $S=\left(U, A, V_{\{0,1\}}, f\right)$.

Definition 2. Let $(F, E)$ be a soft set over the universe $U$ and $u \in U$. A parameter cooccurrence set of an object $u$ can be defined as

$$
\operatorname{coo}(u)=\{e \in E: f(u, e)=1\} .
$$

Obviously, $\operatorname{Coo}(u)=\{e \in E: F(e)=1\}$.

Definition 3. Let $(F, E)$ be a soft set over the universe $U$ and $u \in U$. Support of an object $u$ is defined by

$$
\operatorname{supp}(u)=\operatorname{card}(\{e \in E: f(u, e)=1\})
$$

Definition 4. Let $(F, E)$ be a soft set over the universe $U$ and $u \in U$. An objects $u$ is said to be maximally supported by a set of all parameters $E$, denoted by $\operatorname{Msupp}(u)$ if

$$
\operatorname{supp}(u)>\operatorname{supp}(v), \forall v \in U \backslash\{u\} .
$$

Based on Definition 4, we can make supported (ranked) ordered objects according their support value as

$$
U_{1}>U_{2}>\cdots>U_{n}
$$

where $U_{i} \subseteq U$ and $U_{i}=\{u \in U: u$ is $i$ - th maximal supported by $E\}$, for $1 \leq i \leq n$.

Thus, $U_{i}$ is a collection of objects in $U$ having the same support, i.e., objects of the same support of are grouped into the same class. Obviously $U=\bigcup_{1 \leq i \leq n} U_{i}$ and $U_{i} \cap U_{j}=\phi$, for $i \neq j$. In other word, a collection of $U / E=\left\{U_{1}, U_{2}, \cdots, U_{n}\right\}$ is a decision partition of $U$, so called cluster decision of $U$.

Definition 5. Let $(F, E)$ be a soft set over the universe $U$ and $A \subset E$. A is said to be indispensable if $U / A=U / E$. Otherwise, $A$ is said to be dispensable.

Based on Definition 5, we can reduce the number of parameters without changing the optimal and sub-optimal decisions.

Definition 6. For soft set $(F, E)$ over the universe $U$ and $A \subseteq E$. A is reduction of $E$ if only if $A$ is indispensable and $\operatorname{supp}_{A}(u)={ }_{A} \operatorname{supp}(v)$, for every $u, v \in U$.

Definition 7. For soft set $(F, E)$ over the universe $U$ and $u \in U$. An object $u$ will be the optimal decision if $u$ is maximally supported by $E$. 
Example 3. As for example, the following will be the co-occurrence objects derived from Table 4.

Table 4. An example of Boolean table of representation of a soft set $(F, E)$.

\begin{tabular}{ccccccccc}
\hline $\boldsymbol{U}$ & $e_{1}$ & $e_{2}$ & $e_{3}$ & $e_{4}$ & $e_{5}$ & $e_{6}$ & $e_{7}$ & $f_{E}\left(p_{i}\right)$ \\
\hline$p_{1}$ & 1 & 1 & 1 & 1 & 0 & 0 & 1 & $\mathbf{5}$ \\
$p_{2}$ & 0 & 1 & 1 & 0 & 0 & 1 & 0 & $\mathbf{3}$ \\
$p_{3}$ & 1 & 0 & 0 & 0 & 1 & 0 & 1 & $\mathbf{3}$ \\
$p_{4}$ & 1 & 0 & 1 & 0 & 1 & 1 & 0 & $\mathbf{4}$ \\
$p_{5}$ & 1 & 0 & 0 & 0 & 0 & 0 & 1 & $\mathbf{2}$ \\
$p_{6}$ & 1 & 0 & 0 & 0 & 0 & 0 & 1 & $\mathbf{2}$ \\
\hline
\end{tabular}

$$
\begin{aligned}
& \operatorname{coo}\left(p_{1}\right)=\left\{e_{1}, e_{2}, e_{3}, e_{4}, e_{7}\right\}, \operatorname{coo}\left(p_{2}\right)=\left\{e_{2}, e_{3}, e_{5}\right\}, \operatorname{coo}\left(p_{3}\right)=\left\{e_{1}, e_{5}, e_{7}\right\}, \\
& \operatorname{coo}\left(p_{4}\right)=\left\{e_{1}, e_{3}, e_{5}, e_{7}\right\}, \operatorname{coo}\left(p_{5}\right)=\left\{e_{1}, e_{7}\right\} \text { and } \operatorname{coo}\left(p_{6}\right)=\left\{e_{1}, e_{7}\right\} .
\end{aligned}
$$

Thus, support of each object is given as follow

$$
\operatorname{supp}\left(p_{1}\right)=5>\operatorname{supp}\left(p_{4}\right)=4>\operatorname{supp}\left(p_{2}\right)=\operatorname{supp}\left(p_{3}\right)=3>\operatorname{supp}\left(p_{5}\right)=\operatorname{supp}\left(p_{6}\right)=2 .
$$

Based from the Definition 5, the partition contain four clusters, i.e.,

$$
\left\{\left\{p_{1}\right\},\left\{p_{4}\right\},\left\{p_{2}, p_{3}\right\},\left\{p_{5}, p_{6}\right\}\right\},
$$

where it is arrange in descending order of support value.

As noted that, the first maximal supported patient is $p_{1}$, where $\operatorname{supp}\left(p_{1}\right)=5$ and as been defined by Definition 7, $p_{1}$ is the optimal decision. $p_{4}$ can be considered as the second maximal supported patient based on the support which the next highest, i.e., $\operatorname{supp}\left(p_{4}\right)=4$. And it is also noted that $p_{2}, p_{3}$ and $p_{5}, p_{6}$ can be the second and third maximal supported patients, since $\operatorname{supp}\left(p_{2}\right)=\operatorname{supp}\left(p_{3}\right)=3$ and $\operatorname{supp}\left(p_{5}\right)=\operatorname{supp}\left(p_{6}\right)=2$, respectively.

To elaborate Definition 6, let $A=\left\{e_{2}, e_{3}, e_{5}, e_{6}, e_{7}\right\}$. Then we will obtain

$$
U / A=\left\{\left\{p_{1}\right\},\left\{p_{4}\right\},\left\{p_{2}, p_{3}\right\},\left\{p_{5}, p_{6}\right\}\right\}=U / E,
$$

and since $E \backslash A=\left\{e_{1}, e_{4}\right\}$, then

$$
\operatorname{supp}_{E \backslash A}\left(p_{1}\right)=\operatorname{supp}_{E \backslash A}\left(p_{2}\right)=\cdots=\operatorname{supp}_{E \backslash A}\left(p_{6}\right)=1 .
$$

Therefore, $A$ is parameter reduction of $E$ and we can now delete attribute $A=\left\{e_{1}, e_{4}\right\}$. Note that, by deleting $A$, we now have $U / A=\left\{\left\{p_{1}\right\},\left\{p_{4}\right\},\left\{p_{2}, p_{3}\right\},\left\{p_{5}, p_{6}\right\}\right\}$, which is still 
the same decision partition as in $U / E$. Also in this case, the maximum supported objects are still maintained. As in $[16,18]$, any set of reduct that conforms to our rule of reduct will still provide the same optimal and sub optimal decisions. By comparing optimal and sub-optimal decision from our proposed technique with baseline technique for normal parameterization reduction, also giving the result of the same the optimal and sub-optimal decisions. Furthermore, our proposed technique confirming that the reduction also provides the right objects for decision making.

\section{Result and Discussion}

After calculating co-occurrences of symptoms in each suspect, Figure 1 lists the detailed results of dispensable symptoms and reduct obtained. The symptoms Difficult Breathing and Lethargic will be removed from the dataset. Thus, we obtain the reduct as a subset of symptoms contains

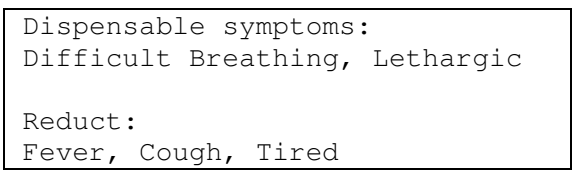

Fig. 1. Reduction of symptoms

After reduction, we can make decisions easily. The decisions are based on the support symptoms of each patient. Patients having the same support are clustered in the same class. There are three clusters decision we can made and they are given in Figure 2.

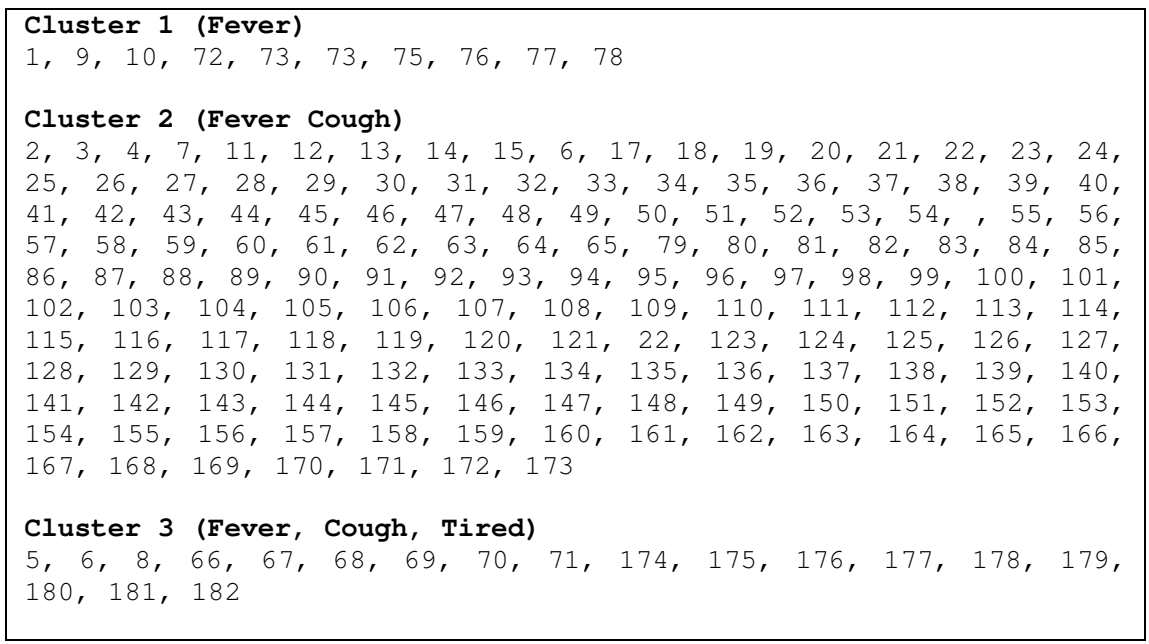

Fig. 2. Decision clusters 
From Figure 2, the numbers of suspects in each cluster and their percentage are given in Table 5 and Figure 3, respectively.

Table 5. Number of suspect and percentage

\begin{tabular}{cccc}
\hline & Fever & Fever, Cough & $\begin{array}{c}\text { Fever, Cough, } \\
\text { Tired }\end{array}$ \\
\hline $\begin{array}{c}\text { Number of } \\
\text { Suspects } \\
\text { Percentage }\end{array}$ & 10 & 154 & 18 \\
\hline
\end{tabular}

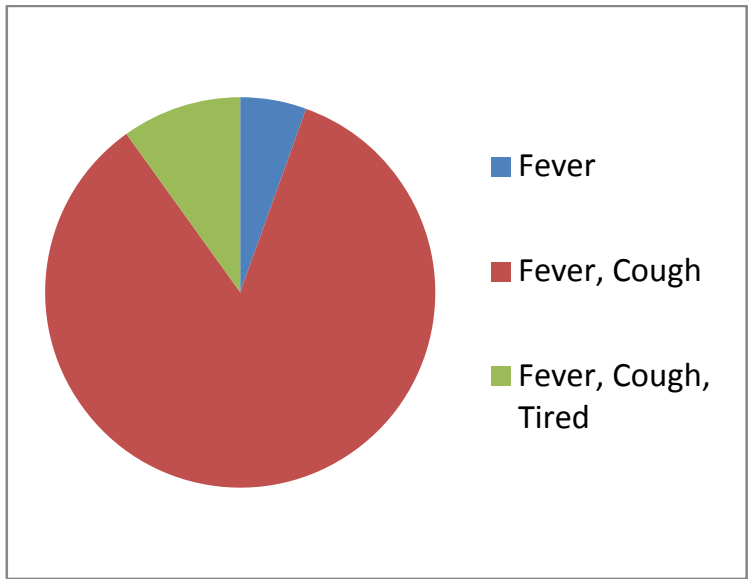

Fig. 3. A 2-D Pie plot of suspect's percentage

With this decision making technique, it is recommended that suspect with higher supported symptoms are indicated having ILI rather than lower supported symptoms.

From Table 5, we can conclude that 18 suspects are highly indicate having ILI, 154 suspects are medium indicate having ILI and 10 suspects are low indicate having ILI.

\section{Conclusion}

We have successfully presented an extended application of our soft set-based decision making for medical decision making. It is applied through a Boolean valued information system representing a dataset of patients suspected ILI (Influenza-Like Illness) taken from University Medical Center, Universiti Tun Hussein Onn Malaysia during MayAugust 2009. Using soft set theory and maximal symptoms co-occurences in patients, we explore how soft set-based decision making technique can be used to reduce the number of dispensable symptoms and further make a correct and fast decision. The result of this work can be used for recommendation of decision making based on the clusters decision captured. It is recommended that suspect with higher supported symptoms are indicated 
having ILI rather than lower supported symptoms. Finally, this technique may potentially contribute to lowering the complexity of medical decision making without loss of original information.

\section{Acknowledgement}

I would like to thank to the Head of Universiti Tun Hussein Onn Malaysia Medical Center for providing the Influenza-Like Illness data. This work was supported by Universiti Malaysia Pahang.

\section{References}

1. M. Michaelis, H.W. Doerr, J. Cinatl Jr. An Influenza A H1N1 Virus Revival Pandemic H1N1/09 Virus. Infection (2009) 37, 381-389.

2. S.M. Burns. H1N1 influenza is here. Journal of Hospital Infection (2009) 73, 200202.

3. K.B. DeGruy. Healthcare Applications of Knowledge Discovery in Databases. Journal of Healthcare Information Management, (2000), 14 (2) 59-69.

4. Caldwell, J. and Y.M. Ram, 1999. Mathematical Modelling: Concepts and Case Studies. Kluwer Academic Publishers, Dordrecht, Netherlands.

5. Li, M.Y., J.S. Muldowney and P.V.N. Driessche, 1999. Global stability of SEIRS model in epidemiology. Can. Applied Mathematics, 7, 409-425.

6. Roberts, M.G. and J.A.P. Heesterbeek, 2003. A new method for estimating the effort required to control an infectious disease. Proc. R. Soc. Lond., 270, 13591364.

7. Chowell, G., C. Castillo-Chavez, P.W. Fenimore, C.M. Kribs-Zaleta, L. Arriola and J.M. Hyman, 2004. Model parameters and outbreak control for SARS. Emerging Infect. Dis., 10, 1258-1263.

8. Gurevich, K.G., 2009. Formal kinetics of H1N1 epidemic. Theor. Biol. Med. Modelling, 6, 23-23.

9. S.A.A. Karim and R. Razali. A Proposed Mathematical Model of Influenza A, H1N1 for Malaysia. Journal of Applied Sciences (2011), 11 (8), 1457-1460.

10. Zhao, Y., Luo, F., Wong, S.K.M. and Yao, Y.Y.: A general definition of an attribute reduct, Proceeding of Second International Conference on Rough Sets and Knowledge Technology, LNAI 4481, 101-108 (2007).

11. D. Molodtsov. Soft set theory-first results, Computers and Mathematics with Applications 37, 19-31, (1999).

12. P.K. Maji, A.R. Roy, and R. Biswas. An application of soft sets in a decision making problem, Computer and Mathematics with Application, (2002), 44, 10771083.

13. D. Chen, E.C.C. Tsang, D.S. Yeung, and X. Wang. The Parameterization Reduction of Soft Sets and its Applications, Computers and Mathematics with Applications, (2005), 49, 757-763.

14. Z. Kong, L. Gao, L. Wang, and S. Li. The normal parameter reduction of soft sets and its algorithm, Computers and Mathematics with Applications, (2008) 56, 30293037. 
15. T. Herawan, A.N.M. Rose and M.M. Deris. Soft set theoretic approach for dimensionality reduction. In D. Ślęzak et al. (Eds.): DTA 2009, Communication of Computer and Information Sciences 64, pp. 180-187, 2009. () Springer-Verlag 2009.

16. A.N.M. Rose, T. Herawan and M.M. Deris. A framework of decision making based on maximal supported sets. In L. Zhang, J. Kwok, and B.-L. Lu (Eds.): ISNN 2010, Part I, Lecture Notes in Computer Science 6063, pp. 473-482, 2010. (C) SpringerVerlag 2010.

17. T. Herawan, R. Ghazali, and M.M. Deris. Soft set theoretic approach for dimensionality reduction. In a special issue of DTA 2009, International Journal of Database Theory and Application, Vol. 3, No. 2, June 2010, Pages 47-60.

18. T. Herawan and M.M. Deris. Soft decision making for patients suspected influenza. In D. Taniar et al. (Eds.): ICCSA 2010, Part III, Lecture Notes in Computer Science 6018, pp. 405-418, 2010. (C) Springer-Verlag 2010.

19. T. Herawan and M.M. Deris. On multi-soft sets construction in information systems. In D.S. Huang et al. (Eds.): ICIC 2009, Lecture Notes in Artificial Intelligence 5755, pp. 101-110, 2009. (Springer-Verlag 2009.

20. Abbas, K., Mikler, A.R. and Gatti R.: Temporal Analysis of Infectious Diseases: Influenza, ACM Symposium on Applied Computing 2005, 267-271.

21. http://pku.uthm.edu.my/ 